Cuestiones de sociología ISSN: 2346-8904

publicaciones@fahce.unlp.edu.ar

Universidad Nacional de La Plata

Argentina

\title{
Políticas sociales y desigualdades en salud: un panorama sobre las personas mayores en el Gran Buenos Aires
}

Findling, Liliana; López, Elsa; Cirino, Estefanía

Políticas sociales y desigualdades en salud: un panorama sobre las personas mayores en el Gran Buenos Aires

Cuestiones de sociología, núm. 22, 2020

Universidad Nacional de La Plata, Argentina

DOI: https://doi.org/10.24215/23468904e091

Atribución no comercial compartir igual (CC BY-NC-SA) 4.0 
Artículos

\title{
Políticas sociales y desigualdades en salud: un panorama sobre las personas mayores en el Gran Buenos Aires
}

\author{
Social policies and health inequalities: an overview of the elderly in Gran Buenos Aires
}

\author{
Liliana Findling \\ Instituto de Investigaciones Gino Germani, Facultad de \\ Ciencias Sociales, Universidad de Buenos Aires, Argentina \\ findling.liliana@gmail.com \\ Elsa López \\ Instituto de Investigaciones Gino Germani, Facultad de \\ Ciencias Sociales, Universidad de Buenos Aires, Argentina \\ lopezelsa4@gmail.com \\ Estefania Cirino \\ Instituto de Investigaciones Gino Germani, Facultad de \\ Ciencias Sociales, Universidad de Buenos Aires, Argentina \\ cirino.estefania@gmail.com
}

\section{ReSUMEN:}

La población argentina muestra un creciente envejecimiento desde los años 70 del siglo pasado con repercusiones en la vida cotidiana a nivel individual, familiar y social. Si bien el país posee una elevada cobertura previsional (90\%), existen notorias heterogeneidades en el acceso a la salud y en la percepción de haberes jubilatorios entre los hogares de diversos niveles socioeconómicos. Este artículo propone los siguientes objetivos: describir las políticas de protección social y de salud destinadas a las personas de 60 años o más que residen en el Gran Buenos Aires en el periodo 2013-2019, y analizar dimensiones referidas a su situación de salud. La metodología se basó en fuentes de información secundarias (leyes y decretos y análisis de encuestas de población). El análisis de los datos permitió identificar desigualdades según jurisdicción, sexo, edad, nivel educativo, de ingresos y trayectoria laboral de las personas mayores. Las transformaciones ocurridas desde 2016 en el ámbito de la protección social muestran una retracción del Estado en su responsabilidad sobre el riesgo social y revelan desconocimiento acerca del impacto del envejecimiento demográfico así como de las necesidades de las personas mayores.

Palabras Clave: Envejecimiento, Desigualdades sociales, Acceso a la salud, Políticas de protección social.

\section{Abstract:}

The Argentine population has been growing older since the 1970s and that has an impact on daily life at the individual, family and social levels. Although the countruy has a high level of social security coverage ( 90 per cent), there are certain marked heterogeneities among households with lower socio-economic levels in terms of access to health care and the perception of retirement benefits. This article proposes the following objectives: to describe social protection and health policies for people aged 60 or over in the period 2013-2019 and, to analyse dimensions related to the health situation of these people residing in Greater Buenos Aires. The methodology is based on the survey of secondary sources (laws and decrees and analysis of population surveys). The data analysis identifies inequalities according to jurisdiction, gender, age, educational level, income and work trajectory among the elderly.The transformations in social protection since 2016 show a retreat of the State in its responsibility for social risk and ignore the impact of ageing as well as the needs of the elderly.

KEYWORDS: Aging, Social inequalities, Access to health, Social protection policies. 


\section{INTRODUCCIÓN}

La población argentina muestra un creciente envejecimiento desde los años 70 del siglo pasado. El descenso de los niveles de fecundidad, las modificaciones en los patrones de morbimortalidad y el aumento de la esperanza de vida contribuyeron al aumento del porcentaje de personas de 60 años o más.

El envejecimiento de la población tiene repercusiones en la vida cotidiana a nivel individual, familiar y social. En lo económico cambian las relaciones entre el ahorro, las inversiones y el consumo, se modifican los mercados de trabajo y se impone un redimensionamiento de las pensiones y las transferencias intergeneracionales. A nivel familiar e individual esos cambios se manifiestan en la composición de la familia y las modalidades de convivencia, lo que afecta las relaciones de intercambio y reciprocidad entre sus miembros. No menos importante es la demanda específica en relación a los servicios de cuidado y de atención de la salud (Laslett, 1995).

Dado que el sistema de protección social es insuficiente para mantener una calidad de vida acorde con las exigencias que plantean las edades avanzadas, una parte significativa de la población mayor debe obtener recursos de diversas redes sociales, fundamentalmente de sus propias familias, cuya participación es un vértice fundamental en el diamante del cuidado (Esping Andersen, 1993; Razavi, 2007). Los vértices restantes, conformados por el Estado, el mercado y las organizaciones no gubernamentales, resultan marginales o complementarios; esto produceuna intensa estratificación en el acceso a servicios y prestaciones que termina por constituirse en un vector de desigualdad (Rodríguez Enríquez y Méndez, 2013).

Hasta hace pocas décadas, las familias ocupaban un lugar preponderante como proveedoras de protección y seguridad, esquema que se ha ido alterando debido a sucesivos cambios sociales (incorporación femenina al mercado de trabajo, redistribución de tareas y de responsabilidades de los miembros de la familia), demográficos (envejecimiento de la población y aumento del "envejecimiento dentro del envejecimiento", disminución de la fecundidad, menor número de miembros del hogar), políticos (modificaciones en las atribuciones del Estado, mercantilización de servicios), y culturales (secularización, individualización, mayor autonomía de las mujeres) (Martín Palomo, 2008; Findling y López, 2015).

De acuerdo al Censo Nacional de Población, Hogares y Viviendas de 2010, en el país residían 5,7 millones de personas mayores de 60 años (el 14,3\% del total de la población). Las proyecciones del Fondo de Población de las Naciones Unidas indican que hacia 2050 el $25 \%$ de la población argentina tendrá 60 años o más (Amadasi y Tinoboras, 2016). Este diagnóstico refleja el promedio nacional, aunque es de destacar que existen diferencias importantes entre diversas regiones del país, como se advierte en la Ciudad Autónoma de Buenos Aires, la jurisdicción más envejecida: en 2010 el 21,7 \% de su población tenía 60 años y más, mientras que la provincia de Buenos Aires mostraba un 14,9\% de personas en esas edades (López, Findling, Lehner y Mario, 2015).

Uno de los rasgos que caracteriza a la población adulta mayor es la feminización debida a la sobremortalidad masculina; a ello se añade su perfil mayormente urbano.

Si bien Argentina posee una elevada cobertura previsional (90\%), existen ciertas heterogeneidades que son notorias en los hogares de estratos socioeconómicos más bajos. Algo más del $50 \%$ de las jubilaciones y pensiones alcanzan solo el haber mínimo, que en diciembre de 2019 era de $\$ 14.000$. Según la ANSES (2019), el sistema cuenta con 6.952.000 beneficiarios contributivos (jubilaciones y pensiones con y sin moratoria), que perciben un haber promedio de $\$ 17.800$. Además, la ANSES otorga 1.700 .000 pensiones no contributivas, con un haber promedio de $\$ 10.350$ (pensión universal para el adulto mayor, pensión por invalidez, madres de 7 hijos, excombatientes, discapacidad).

Conocer las desigualdades sociales relativas al acceso a la salud de las personas mayores permite avanzar en la identificación de los condicionantes y determinantes de la situación de esta población. Para ello es necesario comprender las condiciones y la dinámica en que se formulan las políticas sociales, cómo inciden las reformas 
en los sistemas de protección social y cómo se redefine el Estado para analizar las acciones específicas para estas personas.

Este artículo - que forma parte de un proyecto aprobado y financiado por la Universidad de Buenos Aires ${ }^{1}$ propone los siguientes objetivos: 1) describir las políticas de protección social y salud destinadas a las personas de 60 años o más en el periodo 2013-2019, y 2) analizar dimensiones referidas a la situación de salud de esas personas.

La metodología se basa en el relevamiento de fuentes secundarias (leyes y decretos, revisión bibliográfica, análisis de encuestas de población, rastreo de diarios nacionales). Se han tenido en cuenta, sobre todo, las encuestas disponibles que consideran a las personas mayores a partir de los 60 años y más, a saber: a) la Encuesta de Protección y Seguridad Social (2015) del Ministerio de Trabajo (ENAPROSS) referida a la población de esa edad que reside en el Gran Buenos Aires (CABA y 24 partidos del Conurbano Bonaerense), b) algunos indicadores de la Encuesta Nacional sobre la Estructura Social (ENES), realizada en el marco del Programa de Investigación sobre la Sociedad Argentina Contemporánea (PISAC), que fue relevada durante 2014/2015. ${ }^{2}$ Además se han descripto algunas dimensiones generales dela 4. ${ }^{a}$ Encuesta Nacional de Factores de Riesgo (2018) del Ministerio de Desarrollo Social y Salud. Vale aclarar que este estudio toma en cuenta la categoría de adulto mayor partir de los 65 años y más. ${ }^{3}$ Se hanconsiderado las siguientes dimensiones: características sociodemográficas, acceso a jubilación, pensiones, cuidados, cobertura de salud, acceso a los servicios de salud y a los medicamentos.

El trabajo se divide en cuatro partes: en la primera se exponen conceptos teóricos referidos al envejecimiento y las políticas sociales así como a las desigualdades en salud; en la segunda parte se describen los cambios ocurridos en las políticas de protección social desde 2013 hasta la actualidad a través del rastreo de normativas sobre previsión social y salud; en la tercerase analiza el resultado de los relevamientos de datos sobre previsión social y acceso a la salud provenientes de encuestas y, a modo de conclusión, en la cuarta parte se resumen un conjunto de consideraciones finales.

\section{Algunos EJes CONCEPtuAles}

\subsection{Sobre el envejecimiento}

El siglo XXI se presenta como el siglo más envejecido de la historia de la humanidad. El proceso de la vejez implica una construcción que está determinada por el contexto social. Durante largo tiempo las sociedades modernas asociaron la vejez a la enfermedad, a lo pasivo, a lo que debía ocultarse. En la década del 60 del siglo pasado los estudios sociales tendieron a incorporar, desde diversas perspectivas, los análisis de curso e historia de vida que acompañan la comprensión de la edad como una variable a considerar en los fenómenos sociales (Gastrón y Oddone, 2008). Anteriormente las investigaciones se concentraban en las edades activas y en los procesos e instituciones de socialización y coincidían en una visión productiva del ser humano respecto del desarrollo de las sociedades capitalistas. Hoy,la definición de vejez desde un punto de vista cronológico resulta incompleta y excluyente (Bazo, 1992); por ello, los estudios de la sociología de la vejez se enfocan en los aspectos biológicos, psicológicos y sociales. El enfoque biológico refiere a la concepción cronológica del envejecimiento, mientras que el psicológico introduce nociones asociadas a la socialización y desocialización en diferentes instituciones y/o grupos. El enfoque social considera que la vejez está atravesada por cuestiones económicas (pérdida progresiva de las funciones), por una dependencia estructural unida directamente a la jubilación y por una trama social relacionada con lo productivo, que se muestra como el origen de la dependencia (Huenchan y Rodriguez-Piñero, 2010). Estas cuestiones traen aparejadas implicancias en el desarrollo de las políticas sociales hacia la vejez ya que, en muchos casos, se asocia la vejez a la pobreza (Casals, 1980). 
Desde la sociología de la vejez se intenta mostrar diferentes modos de envejecer comprendiendo que su construcción es diversa y que la historia de vida de los actores marca distintos modos de ser en el mundo y con el mundo (Rada Shultze, 2018).

Pérez (2000) considera que es necesario superar la mirada que vuelve uniforme la experiencia de la vejez para develar la heterogeneidad de las formas de envejecer. Así, el envejecimiento, en tanto proceso históricosocial o individual, es dinámico, heterogéneo y particularmente contextualizado (Tamer, 2008).

El enfoque del envejecimiento activo (EA) que implementó la Organización Mundial de la Salud busca subrayar los aspectos positivos del envejecimiento, diferenciándose de conceptos como los de envejecimiento saludable, satisfactorio, competente y productivo (Alfama y Cruells, 2016). Si bien algunos académicos e investigadores de la vejez han utilizado el concepto de EA focalizándose en el contexto económico, específicamente en el mercado de trabajo, la OMS lo utiliza para referirse a la salud en su concepción amplia de condición de bienestar biológico, psicológico y social. Las definiciones acerca de lo que se entiende como envejecimiento activo, saludable o exitoso en la literatura académica son diversas y los estudios datan de mediados del siglo XX. El discurso sobre el EA y las políticas relacionadas a éste se han insertado en los discursos de los organismos internacionales y se han transformadoen piedras angulares de un nuevo marco interpretativo y de acción relevante en el proceso de envejecer,que busca construir nuevos sujetos (Pla y Pérez Salanova, 2016). No obstante, ese discurso se ha mostrado como un concepto polisémico, ambiguo, cambiante y de contornos difusos.

Una de las posturas establece que el EA se encuentra atravesado por tres marcas: la calidad de vida y el autodesarrollo, los derechos humanos, y la sostenibilidad del sistema. La primera concibe al envejecimiento como un logro histórico de la humanidad a nivel demográfico: cada vez las poblaciones envejecen más y más rápido, las personas viven más, y, en algunos casos, en mejores condiciones que antes. Esta visión establece la importancia de actuar a lo largo del ciclo vital de las personas, a través de diferentes políticas y programas, para prevenir la dependencia y promover oportunidades de salud y cuidados, haciendo hincapié en el entorno social. La segunda marca refiere a las personas mayores y su rol como ciudadanos y sujetos de derechos, por lo que se propone promover la autonomía, dignidad, seguridad y cuidado. Y la última marca implica una preocupación por la sostenibilidad de los sistemas de bienestar, seguridad social, salud y cuidados. Propone reducir los costos vinculados al envejecimiento, promover el pleno empleo en todas las edades, alargar la vida laboral y restringir la jubilación temprana (Alfama y Cruells, 2016).

\subsection{Sobre las políticas sociales y las desigualdades}

Para analizar las políticas destinadas a las personas mayores se deben considerar dos niveles: el micro, que se centra en aspectos como la provisión para usuarios específicos, respuestas a problemas particulares o gestión de los mismos, y, en segundo término, el macro, que intenta caracterizar y comparar sistemas de bienestar entre sociedades referidos a la organización de las respuestas a las necesidades sociales de un país (Adelantado Gimeno, 2017).

Las políticas sociales constituyen el ámbito en el que se resuelve la cohesión social y el poder de integración; más específicamente, aluden a un conjunto de servicios sociales y normas institucionales prestadas por el Estado (jubilaciones y pensiones), y a instancias de gestión y/o control (obras sociales, asignaciones familiares, seguros de desempleo) (Danani, 1998). Estas políticas deben enmarcarse en un espacio crítico y son el resultado de la politización del ámbito de la reproducción. Las políticas sociales reflejan cómo una determinada sociedad se acerca o se aleja del reconocimiento de las necesidades de su población y cuál es su capacidad de protección (Grassi, 2003).

¿A qué se refiere la capacidad de protección social? Es el alcance de las necesidades de prestaciones y servicios que brinda la seguridad social. Las necesidades pueden ser: a) cuantitativas: cuánto y hasta dónde son satisfechas, para qué sectores de población, y b) cualitativas: calidad y garantías provistas por el sistema 
(Danani e Hintze, 2011). En ese sentido, la protección social es parte de las condiciones generales de la reproducción de la fuerza de trabajo y, por lo tanto, de la reproducción de la vida del conjunto de la población en una sociedad capitalista (Danani y Hintze, 2014).

Los aspectos que condicionan el bienestar o malestar de las personas de más edad son de diversa índole. Las desigualdades observables en las condiciones de vida de las personas dan lugar a diferencias injustas que se van profundizando con el tiempo. El impacto de las repetidas crisis económicas convierte a la desocupación y los bajos ingresos en fenómenos de larga duración, que se extienden desde los aspectos materiales hasta los simbólicos.

En lo que se refiere a la salud, las dimensiones socioculturales y económicas (cobertura de salud, nivel de ingresos, distancias sociales y culturales con los profesionales de la salud, dificultades para el acceso a los centros de atención (Corin, 1996) juegan un papel fundamental en las desigualdades, porque condicionan una percepción diferencial de síntomas y enfermedades, lo cual favorece o dificulta el acceso a los servicios de salud.

Conocer cómo se generan las políticas de salud y de protección social es un eslabón imprescindible para analizar la persistencia delas desigualdades de las personas mayores. A su vez, analizar estas desigualdades permitiría elaborar políticas y estrategias de intervención, identificar los condicionantes y determinantes del estado de salud de población adulta mayor.

En numerosos estudios (Borrell y Benach, 2003; Barradas Barata et al., 2002; Macintyre, Hunt y Sweeting, 1996; Barbosa Da Silva y Berti Azevedo Barros, 2002; Gwatkin, 2000) se ha señalado el papel que desempeñan el sexo, la edad, la etnia y la situación sociocultural y económica de las personas en la generación de las desigualdades en salud (cobertura, nivel educacional, distancias socioculturales y espaciales a los centros de atención de la salud, redes familiares y sociales, entre otras dimensiones). Muchos de esos estudios han centrado la atención en las diferentes maneras en las cuales varones y mujeres perciben las enfermedades y se acercan a los servicios de salud. Algo similar ocurre con las distintas etapas de la vida de las personas; a medida que avanza la edad, algunas dolencias adquieren preeminencia y otras pasan a segundo plano.

La Organización Panamericana de la Salud (OPS, 1999) señala que el concepto de inequidad se ha considerado sinónimo del concepto de desigualdad, pero es fundamental distinguirlos. Mientras desigualdad implica diferencia entre individuos o grupos de población, inequidad representa la calificación de esta diferencia como injusta. Cierto es que no todas las desigualdades son injustas, aun cuando toda inequidad supone una desigualdad injusta.

Así, la igualdad es un concepto empírico que facilita el análisis descriptivo, al tiempo que la equidad constituye un imperativo ético asociado con principios de justicia social; en consecuencia, un concepto normativo (Barbosa da Silva et al., 2002). Al respecto, estos autores distinguen entre desigualdades naturales y sociales. Las naturales ocurren como diferencias entre individuos como el sexo, la raza y la edad, y las sociales se originan en la ocupación de distintas posiciones en la organización social del proceso de producción. De este modo, determinan un diferente acceso a bienes de escasa disponibilidad. Las desigualdades naturales se convierten en sociales cuando son utilizadas por una sociedad como criterio para la asignación de roles sociales. Así, una desigualdad social se convierte en injusta cuando surge de un juicio de valor que aparece en el concepto de inequidad.

En la misma línea, Borrell y Benach (2003) consideran que desde el punto de vista de la estructura social el concepto de desigualdad hace referencia a las diferencias existentes en el acceso a los recursos que tienen las personas tanto en calidad como en cantidad. Su estudio es importante porque el acceso a los recursos se relaciona con las condiciones de vida de las personas.

Otros autores como Sen (2002) plantean que la equidad en salud es inevitablemente multidimensional. Existen factores que intervienen en el logro de la salud que van más allá de la prestación y distribución sanitaria: predisposiciones genéticas, ingresos individuales, hábitos alimentarios, estilos de vida, entorno 
epidemiológico y condiciones de trabajo. Por ello la equidad en salud no se puede analizar solo en términos de la distribución de la atención sanitaria.

Es interesante destacar el abordaje de Rohlfs et al.(2000) sobre género y salud porque aclara que este concepto no es una variable. El género se constituye a través de las relaciones sociales, roles y expectativas ligados a las atribuciones femeninas y masculinas, que no son fijas, cambian según la cultura y a lo largo de la historia (Scott, 1988). A medida que se puedan conocer mejor los determinantes biológicos y sociales, tanto de la salud como de la enfermedad, se podrá avanzar en la posibilidad de diseñar programas y políticas sanitarias para mejorar la calidad de vida, evitar y minimizar los efectos de las enfermedades y eliminar las inequidades en la salud de mujeres y hombres. Según Alleyne (2000), la división en ámbitos de acción masculinos y femeninos marca diferencias sustantivas tanto en los riesgos y necesidades sociales y biológicas, como en las oportunidades, recursos y contribuciones para la salud.

A partir del surgimiento de la epidemiología en Europa y de la aparición de la Medicina Social a fines de 1970, se elaboraron abordajes que permitieron delinear perfiles epidemiológicos de clase. Se retomó el interés por las desigualdades en salud a partir del informe Black en 1980, elaborado en Gran Bretaña durante el gobierno de Thatcher como respuesta a las políticas de corte neoliberal. En los años 90 se amplió la preocupación de investigadores y agencias internacionales por la inequidad y se incorporaron otros indicadores al análisis: esperanza de vida, incapacidad, muertes evitables y morbilidades específicas. Los estudios pasan a ser relevantes como reflejo de los cambios estructurales en las economías, la ocupación y el estatus. Así, se comienzan medir las desigualdades absolutas y relativas, de efecto e impacto y brutas y moderadas (Kunst, 1997). La expresión determinantes sociales resume el conjunto de factores sociales, políticos, económicos, ambientales y culturales que ejercen gran influencia en el estado de salud (OMS, CDSS, 2008).

El agravamiento de las crisis socioeconómicas en la Argentina convierte a la desocupación y los bajos ingresos en fenómenos de larga duración, que se extienden desde aspectos materiales a otras dimensiones sociales y agrava las desigualdades existentes.

\section{UN RASTREO DE LAS POLÍTICAS: LOS VAIVENES DE LA PREVISIÓN SOCIAL Y LA SALUD 2013-2019}

Es preciso tener en cuenta la orientación neoliberal de las reformas de la década de 1990, que condujo a la transformación de los derechos del sujeto. Esas políticas se basaron en una mirada de la vida social tendiente a la búsqueda de acciones hacia el propio interés de las personas. En la implementación de las reformas, aún hoy siguen vigentes los itinerarios de la modernización que provocaron un proceso de individualización en el marco de un nuevo capitalismo (Grassi, 2003).

Ya en el siglo XXI se podría denominar contrarreforma del modelo neoliberal de los 90 a un conjunto de políticas estatales encaradas desde el año 2003 (Danani y Hintze, 2011). Se priorizó el combate contra la pobreza y la revisión de las políticas sugeridas por las agencias multilaterales en la región. Si bien las medidas generales de reordenamiento económico (derogación de la reforma laboral y negociación colectiva para salarios) mejoraron considerablemente los niveles de empleo y pobreza, poco se avanzó en disminuir la desigualdad estructural existente entre las provincias, así como la inequidad en la distribución del ingreso (Findling, 2012). La ampliación del gasto social en el PBI (especialmente a partir de 2007) crece en seguridad social, en prestaciones no contributivas y en las políticas de tipo universal (educación, salud) (Messina, 2015).

El impacto de las moratorias previsionales (creadas en 2005 y ampliadas en 2014) llamadas Plan de Inclusión Previsional motivó el aumento considerable de la cantidad de personas en edad de jubilación que no contaban con suficientes años de aportes. Dicha medida logró una cobertura de la población pasiva cercana al $95 \%$, que también puede explicarse por la sanción de la Ley No 26.844 del Régimen Especial de Contrato de Trabajo para el Personal de Casas Particulares promulgada en 2013 y por una mayor incorporación 
de trabajadores monotributistas (López et al., 2015; Messina, 2015). Con la mayor cobertura del Plan de Inclusión Previsional disminuyó la participación laboral de las personas mayores perceptoras de este beneficio, especialmente entre aquellos menos escolarizados y entre las mujeres. En cambio, el empleo creció entre los no perceptores (principalmente varones menores de 65 años), y mujeres y varones con mayor nivel educativo (Sala, 2012).

A partir de 2016, durante la presidencia de Macri, con una impronta más cercana a políticas de corte neoliberal, se instrumentaron varias medidas que modificaron el rumbo del anterior gobierno en materia de previsión social. En este sentido, una de las primeras acciones fue la reglamentación de Ley No 27.260/16,que creó:

a) El Programa Nacional de Reparación Histórica para Jubilados y Pensionados que reconoce, en parte, errores en los montos de jubilaciones y propone una actualización para aquellos que hayan iniciado o no un juicio por reajuste de haberes.

b) La Pensión Universal para el Adulto Mayor (PUAM), un beneficio de carácter vitalicio y no contributivo para las personas mayores de 65 años que no perciban una jubilación. El monto asciende al $80 \%$ del haber mínimo establecido por ANSES y el beneficiario puede seguir trabajando en el mercado formal; sus aportes podrán computarse para obtener una jubilación a futuro. Este beneficio incluye la cobertura de PAMI, no es pensionable e impone límites para las personas migrantes.

c) La moratoria previsional: se establecen restricciones para quienes pretenden obtener el beneficio jubilatorio y no cuenten con los años de aportes necesarios, estableciendo diferencias entre varones y mujeres (Sosa y Mársico, 2018).

A partir de la Ley No 27.426 de Reforma Previsional, aprobada en un marco sumamente conflictivo a fines de 2017, se modifica el cálculo de los haberes, aunque su aprobación legislativa suscitó situaciones controversiales. Esta ley utiliza como base el 70 \% de inflación (de acuerdo a la medición del Índice de Precios al Consumidor del INDEC (IPC) y el $30 \%$ de la variación de la Remuneración Imponible Promedio de los Trabajadores Estables (RIPTE). Ambos indicadores (IPC y RIPTE) están referidos a un trimestre y se aplican con dos períodos de atraso. Estas modificaciones incluyeron a 17 millones de beneficiarios (jubilados, pensionados, asignaciones familiares, asignación universal por hijo, pensiones no contributivas, veteranos de guerra) (González y González Etkin, 2017). También se instituye una garantía adicional para los haberes jubilatorios equivalente al $82 \%$ del salario mínimo vital y móvil en el caso de aquellos jubilados que hayan realizado 30 años de aportes. En diciembre de 2015 la cifra de pensiones y jubilaciones por moratoria alcanzó a 3.377.144 de personas. Entre enero de 2016 y junio de 2019 ese número disminuyó a 653.290 beneficios (Boletín Estadístico de la Seguridad Social, 2015 y 2018). Entre enero de 2017 y principios de 2019 se jubilaron 120.000 personas a través de la PUAM. De estos, el $74 \%$ de los beneficiarios son varones, situación que se explica, aunque con mayores restricciones, en que la moratoria para las mujeres sigue en vigencia. Mediante la resolución del 17 de abril de 2019 publicada en el Boletín Oficial, la ANSES impuso nuevos requisitos para acceder a la PUAM, ya que se estima realizar una evaluación a los mayores de 65 años para determinar si, por su situación de ingresos y patrimonial, pueden recibir el beneficio (Roqué et al., 2019).

En lo que se refiere a la situación de salud de las personas mayores, el Instituto Nacional de Servicios Sociales para Jubilados y Pensionados (INSSJyP), más conocido como PAMI, cubre a casi 5 millones de personas. Se financia tanto con aportes y contribuciones de los trabajadores activos y de sus empleadores como con aportes de los trabajadores retirados y contribuciones del Tesoro Nacional. Aquellos afiliados que prefieran cambiar de PAMI a otra cobertura de salud deben transitar por un arduo proceso burocrático. Pese a que es posible cambiar de obra social (el listado para optar por otra entidad está disponible en el Registro de Agentes del Sistema Nacional del Seguro de Salud), la oferta de pases es limitada y los rechazos son frecuentes. Por otra parte, las obras sociales de origen imponen trabas a los jubilados para permanecer como usuarios de la 
institución en la que se han desempeñado en la etapa activa y se debe apelar a mecanismos legales para su reconocimiento.

A partir de 2016 PAMI implementó varias acciones tendientes a restringir beneficios con el argumento de combatir abusos y reducir gastos. En abril de 2016 informó sobre un nuevo listado de remedios en el que cerca de 160 medicamentos pasaban de la cobertura del $100 \%$ a la del $50 \%$. La medida se justifica, según las autoridades, por la "baja utilidad clínica que poseen dichas drogas" (Riera, 2016). Así, de diez unidades que se otorgaban de forma gratuita, en la actualidad solo se otorgan cuatro. Para obtener este beneficio se deben cumplir con determinadas condiciones: cobrar menos o el equivalente a 1,5 haberes previsionales mínimos, no poseer más de una propiedad, un auto o embarcación de no más de diez años de antigüedad y no contar con cobertura de saludadicional a través de una empresa de medicina prepaga.

Además, se modificó el trámite para las solicitudes que deben realizarse online en el Sistema de Medicamentos sin cargo, y, en el caso de solicitar más de cuatro unidades por persona, el PAMI efectúa una evaluación. En todos los casos se requiere una "Declaración Jurada y se otorga el beneficio basado en criterios de vulnerabilidad que permitan priorizar aquellas solicitudes de mayor urgencia” (INSSJ-PAMI, 2017). Esta modalidad implica una serie de trámites de orden burocrático para los afiliados en los que no se especifican los tiempos de evaluación por parte del organismo. Como atenuante se estipulan excepciones para las personas censadas en relevamientos de barrios populares (por certificado expedido por la Administración Nacional de la Seguridad Social o cruce de padrón); que vivan en zonas rurales determinadas por el INSSJP; que se encuentren en tratamiento de diálisis, tratamiento activo de cáncer, quimioterapia o radioterapia, tratamientos de oxigenoterapia permanente, para los insulinorrequirientes, electro dependientes o para personas con discapacidad que cuenten con el correspondiente Certificado Único de Discapacidad (CUD). Además se ha creado recientemente la Receta Electrónica que reemplaza al recetario en papel,por lo que el afiliado debe contar con un mail y/o celular al que se le envían las recetas de medicamentos previamente solicitadas a los médicos de cabecera.

Cabría preguntarse si las condiciones de acceso ya constituyen de por sí una limitación al servicio que tenían los afiliados para obtener medicamentos considerando que las personas mayores no cuentan con la formación necesaria para el uso de tecnologías digitales. PAMI señala que las gestiones las puede realizar el afiliado o alguien de su familia que esté a cargo (INSSJ-PAMI, 2017), lo que origina más responsabilidades en el ámbito familiar (tareas que habitualmente recaen entre las mujeres).

\section{Un panorama sociodemográfico de las personas mayores en el Gran Buenos Aires}

Se describen las principales características sobre la situación de las personas mayores a partir de los datos de la ENAPROSS (2015) que han sido reprocesados para una submuestra de las personas de 60 años o más que residían en la Ciudad Autónoma de Buenos Aires (CABA) y el Conurbano de Buenos Aires (Conurbano). ${ }^{4}$

Casi la totalidad (n: 2.035) de los encuestados proviene, en partes iguales, de la CABA y del Conurbano, con una representación porcentual mayor de mujeres ( $58 \%$ ), que se explica por la sobremortalidad masculina observable en las edades estudiadas (60 años o más). El mayor peso relativo de las personas encuestadas se encuentra entre los 66 y los 75 años (el $40 \%)$. En cuanto al nivel de instrucción, predominan las personas con escolaridad secundaria incompleta o menos. Tres cuartas partes de los encuestados viven en hogares con dos personas o más y el $55 \%$ de ellos convive con su pareja: especialmente los varones y los más jóvenes, debido a la viudez.

Más de la mitad de los encuestados atiende su salud a través del PAMI ( $53 \%$ ) y casi un tercio lo hace mediante otra obra social (30\%). El hospital público es utilizado por el $9 \%$ y las prepagas por el $7 \%$. Estas cifras sufren alteraciones al analizar la distribución por región, sexo, edad e instrucción, ya que la cobertura del PAMI es mayormente utilizada por los residentes del Conurbano, las mujeres, las personas de 75 años o más 
y aquellas con niveles inferiores al secundario completo. El $5 \%$ de las personas afirma contar con Certificado de Discapacidad

En cuanto a sus trayectorias laborales, casi el total de la muestra (95\%) indica que alguna vez ha trabajado.

En lo que se refiere a la condición de jubilación, el $74 \%$ percibe jubilación; esta cifra es algo superior en CABA que en los 24 partidos del Conurbano. Un $10 \%$ no accedió a este beneficio pero planifica iniciar los trámites para jubilarse, mientras que el $16 \%$ restante no tiene jubilación y no piensa tenerla.

Entre las personas que tienen jubilación, las mujeres exhiben un porcentaje más alto que los varones: casi el $80 \%$, contra dos tercios de los varones; las diferencias crecen a medida que avanza la edad (Cuadro No 1).

CUADRO No 1

Condición de jubilación por región, sexo, edad y nivel de instrucción en \%

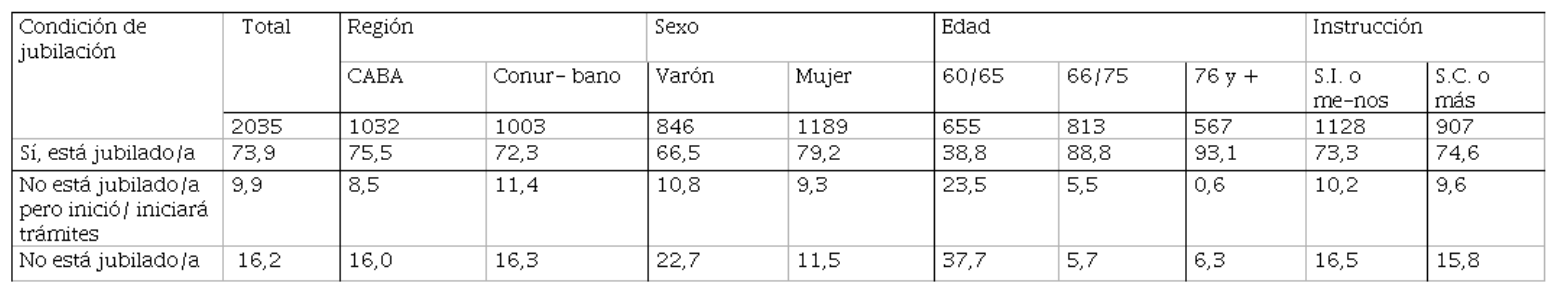

Fuente: Procesamiento propio con base en ENAPROSS II, 2015.

El $58 \%$ de las personas que componen la muestra se han jubilado por años de trabajo y/o aportes y el $42 \%$ por una moratoria cercana al momento de la encuesta. Es de destacar que los jubilados por aportes predominan entre los habitantes de la CABA (65\%) respecto de los del Conurbano (49\%), y entre los varones $(81 \%)$ respecto delas mujeres $(44 \%)$.

También es mayor el porcentaje de jubilados por aportes entre las personas con mayor nivel de instrucción (69\%). Lo mismo ocurre a medida queaumenta la edad, dato esperable, ya que en el tramo inicial, de 60 a 65 años, hay más personas que no han alcanzado la edad jubilatoria ni los años de aportes.

En cuanto a aquellos que accedieron a la jubilación mediante la moratoria, se detecta que los porcentajes son más elevados en el Conurbano y es un beneficio que favorece a las mujeres, a los más jóvenes y a los de menor instrucción.

$\mathrm{Al}$ analizar la modalidad de jubilación por quintiles de ingreso se advierte que la situación económica juega un rol fundamental. Tanto en CABA como en el GBA el porcentaje de personas que perciben una jubilación/ pensión disminuye considerablemente entre los que se ubican en los menores quintiles de ingresos y, a su vez, aumenta el número de personas que accede a este derecho a través de la moratoria. Un panorama similar se observa entre los residentes de los 24 partidos del Conurbano, aunque la brecha es mayor: los porcentajes de acceso al beneficio por moratoria son más elevados comparados con CABA entre los de menores ingresos. Estos datos refuerzan la desigualdad por nivel socioeconómico, ya que se presupone que entre estos sectores socioeconómicos solo se ha podido tener acceso (por moratoria) al haber mínimo.

La edad promedio jubilatoria es de 64 años y varía en pocos años según el grupo de edad, el sexo y la región, aumentando a medida que crece la edad de las personas. La mediana se ubica en 65 años y muestra mínimas diferencias según sexo.

Uno de cada cinco encuestados ha recibido una pensión (dicho beneficio aumenta entre las personas del Conurbano, entre las mujeres, las personas de mayor edad, las que tienen un nivel de instrucción menor al secundario y las que viven solas, probablemente viudas). 
Si se analizan algunos indicadores sobre la salud relevados en la ENAPROSS, puede observarse que el $86 \%$ de los encuestados realizó consultas médicas en los últimos 12 meses, y este porcentaje es algo más frecuente en las mujeres y en los que tienen mayor nivel de instrucción. Estos valores aumentan con la edad (Cuadro $\mathrm{N}^{\circ} 2$ ).

\section{CUADRO $\mathrm{N}^{\circ} 2$}

Realización dealguna consulta médica en los últimos 12 meses (se excluyen consultas odontológicas) por región, sexo, edad y nivel de instrucción en \%

\begin{tabular}{|l|l|l|l|l|l|l|l|l|l|l|}
\hline & Total & \multicolumn{2}{|l|}{ Región } & \multicolumn{2}{l|}{ Sexo } & \multicolumn{2}{l|}{ Edad } & \multicolumn{2}{l|}{ Instrucción } \\
\hline & & CABA & $\begin{array}{l}\text { Conur- } \\
\text { bano }\end{array}$ & Varón & Mujer & $60 / 65$ & $66 / 75$ & $76 \mathrm{y}+$ & $\begin{array}{l}\text { S.I. o } \\
\text { menos }\end{array}$ & $\begin{array}{l}\text { S.C. o } \\
\text { más }\end{array}$ \\
\hline N & 2035 & 1032 & 1003 & 846 & 1189 & 655 & 813 & 567 & 1128 & 907 \\
\hline Total & 100,0 & 100,0 & 100,0 & 100,0 & 100,0 & 100,0 & 100,0 & 100,0 & 100,0 & 100,0 \\
\hline Sí & 86,0 & 87,9 & 84,0 & 82,9 & 88,2 & 79,1 & 88,4 & 90,5 & 84,1 & 88,3 \\
\hline No & 14,0 & 12,1 & 15,9 & 17,1 & 11,7 & 20,9 & 11,4 & 9,5 & 15,9 & 11,6 \\
\hline
\end{tabular}

FUENTE: Procesamiento propio con base en ENAPROSS II, 2015.

El $40 \%$ de los encuestados manifiesta no tener problemas de salud de larga duración ( $43 \%$ en el Conurbano y $36 \%$ en la CABA), cifras que podrían atribuirse a la diversa composición por edad de la población de ambas jurisdicciones, con mayor preponderancia de personas de edad más avanzada en la Capital. De acuerdo a lo esperado, los que declaran no tener problemas de salud de larga duración son más numerosos ( $48 \%$ ) entre los 60 y los 65 años que en los de 76 años o más (32\%).

También como consecuencia de esa distribución por edad de la población, el $32 \%$ afirma requerir más de un tipo de tratamiento o ayuda ( $41 \%$ en la CABA contra el $23 \%$ en el Conurbano de Buenos Aires), hecho que crece cuando aumenta el nivel de instrucción. Una cuarta parte de los encuestados declara necesitar solo medicación ( $21 \%$ en la CABA y $28 \%$ en el Conurbano) (Cuadro $\mathrm{N}^{\circ} 3$ ).

\section{CUADRO $\mathrm{N}^{\circ} 3$}

Problemas de salud de larga duración y tipo de tratamiento o ayuda requeridapor región, sexo, edad y nivel de instrucción en \%

\begin{tabular}{|c|c|c|c|c|c|c|c|c|c|c|}
\hline & \multirow[t]{2}{*}{ Total } & \multicolumn{2}{|l|}{ Región } & \multicolumn{2}{|l|}{ Sexo } & \multicolumn{3}{|l|}{ Edad } & \multicolumn{2}{|c|}{ Instrucción } \\
\hline & & $\mathrm{CABA}$ & Conur-bano & Varón & Mujer & $60 / 65$ & $66 / 75$ & $76 y+$ & $\begin{array}{l}\text { S.I. o } \\
\text { me-nos }\end{array}$ & $\begin{array}{l}\text { S.C. o } \\
\text { más }\end{array}$ \\
\hline N & 2035 & 1032 & 1003 & 846 & 1189 & 655 & 813 & 567 & 1128 & 907 \\
\hline Total & 100,0 & 100,0 & 100,0 & 100,0 & 100,0 & 100,0 & 100,0 & 100,0 & 100,0 & 100,0 \\
\hline $\begin{array}{l}\text { Requiere más de } \\
\text { un tipo de } \\
\text { tratamiento o } \\
\text { ayuda }\end{array}$ & 31,9 & 40,9 & 22,6 & 32,3 & 31,6 & 25,7 & 32,7 & 37,9 & 29,2 & 35,4 \\
\hline $\begin{array}{l}\text { Solo requiere } \\
\text { medicación }\end{array}$ & 24,5 & 20,5 & 28,7 & 22,8 & 25,7 & 22,9 & 25,2 & 25,5 & 26,6 & 21,9 \\
\hline $\begin{array}{l}\text { Solo necesita } \\
\text { controlo algunas } \\
\text { ayudas }\end{array}$ & 3,9 & 2,7 & 5,2 & 4,1 & 3,8 & 3,5 & 3,7 & 4,8 & 4,0 & 3,8 \\
\hline $\begin{array}{l}\text { No padece } \\
\text { problemas de } \\
\text { salud de larga } \\
\text { duración }\end{array}$ & 39,7 & 35,9 & 43,5 & 40,8 & 38,9 & 47,9 & 38,4 & 31,8 & 40,2 & 38,9 \\
\hline
\end{tabular}

FUENTE: Procesamiento propio con base en ENAPROSS II, 2015.

El $82 \%$ de las personas con problemas de salud de larga duración expresan que tienen todo lo que necesitan para atender esa situación, más frecuentemente en la CABA que en el Conurbano (86\% contra $77 \%$ ), lo que demuestra que el aumento del porcentaje se manifiesta a medida que lo hace la edad y el nivel de instrucción. 
Los que dicen no recibir nada de lo que necesitan suman el $6 \%$, atribuible a que más de la mitad de ellos solo dispone de una parte de la cobertura de salud para esos requerimientos.

La mayor parte de los costos que insumen el tratamiento de salud, la medicación o el cuidado es provisto, en primer lugar, por el PAMI (38\%), en segundo lugar por la persona encuestada, un familiar o un amigo (33\%), y, por último, por la prepaga o mutual o la obra social (12\% cada una).

En la cobertura del PAMI se aprecian diferencias importantes entre la que se otorga en el Conurbano de Buenos Aires (50\%) y la recibida por los habitantes de la CABA (29\%). Esto es visible también tanto en la distribución por sexo $-42 \%$ de las mujeres contra $33 \%$ de los varones-, como en la división por edad - $25 \%$ en las personas de 60 a 65 años y $41 \%$ en las de 76 o más-. Además, ocurre algo similar en la discriminación según el nivel de instrucción: 51 \% para los menos educados y $24 \%$ para el resto. En contraste, la cobertura de las prepagas y obras sociales crece entre los que residen en CABA respecto de los habitantes del Conurbano ( $17 \%$ versus $7 \%)$, y entre los más instruidos ( $22 \%$ contra $4 \%)$. Entre aquellos que no reciben la medicación, la cobertura responde solo en una parte del total (Cuadro $\left.\mathrm{N}^{\circ} 4\right)$.

\section{CUADRO $\mathrm{N}^{\circ} 4$}

De quien recibe la medicación, tratamiento, cuidado o ayuda por región, sexo, edad y nivel de instrucción en \%

\begin{tabular}{|c|c|c|c|c|c|c|c|c|}
\hline & \multirow[t]{2}{*}{ Total } & \multicolumn{2}{|c|}{ Región } & \multicolumn{2}{|l|}{ Sexo } & \multicolumn{3}{|l|}{ Edad } \\
\hline & & CABA & $\begin{array}{l}\text { Conur- } \\
\text { bano }\end{array}$ & Varón & Mujer & $60 / 65$ & $66 / 75$ & $76 \mathrm{y}+$ \\
\hline $\mathrm{n}$ & 1006 & 567 & 439 & 408 & 598 & 252 & 420 & 334 \\
\hline Total & 100,0 & 100,0 & 100,0 & 100,0 & 100,0 & 100,0 & 100,0 & 100,0 \\
\hline $\begin{array}{l}\text { La persona con el problema de } \\
\text { salud, de un familiar o un amigo }\end{array}$ & 33,3 & 38,8 & 26,2 & 34,8 & 32,3 & 38,5 & 30,7 & 32,6 \\
\hline PAMI & 38,1 & 29,1 & 49,7 & 32,6 & 41,8 & 25,0 & 41,0 & 44,3 \\
\hline Obra social & 11,7 & 11,5 & 12,1 & 14,2 & 10,0 & 15,9 & 10,5 & 10,2 \\
\hline Prepaga o mutual & 12,3 & 16,6 & 6,8 & 14,0 & 11,2 & 12,7 & 14,8 & 9,0 \\
\hline $\begin{array}{l}\text { Hospital público/ Centro de } \\
\text { salud/INCLUIR SALUD(ex } \\
\text { PROFE) }\end{array}$ & 3,9 & 3,4 & 4,1 & 3,7 & 3,6 & 7,6 & 2,7 & 2,1 \\
\hline NS/NC & 0,9 & 0,7 & 1,1 & 0,7 & 1,0 & 0,4 & 0,5 & 1,8 \\
\hline
\end{tabular}

FUENTE: Procesamiento propio conbase en ENAPROSS II, 2015.

En relación con el consumo de los medicamentos para las personas mayores, su costo se ha incrementado notablemente debido al precio de los insumos importados durante estos últimos años. Según el CEPA, el incremento de los medicamentos desde el año 2015 hasta mayo de 2019 fue de un 298 \%, y algunos medicamentos esenciales alcanzaron a más del $700 \%$ (sobre todo aquellos para enfermedades cardiovasculares, del sistema circulatorio o de tumores). Mientras tanto, el monto de las jubilaciones mínimas ha crecido a un ritmo más lento: desde mayo de 2015 hasta abril de 2019 solo han aumentado un $172 \%$ (Roqué et al., 2019). Estas cifras cobran relevancia dadas las restricciones impuestas por el PAMI, que ya fueron descriptas y que obstaculizan el acceso a medicamentos.

A partir de los resultados de la ENES-PISAC (2014/15), puede detectarse que la distribución de la cobertura de salud es bastante similar -en ambas jurisdicciones-a los datos de la ENAPROSS, ya que PAMI es la cobertura más indicada por los entrevistados (56\%) y algo más de la cuarta parte menciona poseer una obra social.

La percepción del estado de salud se utiliza en muchos estudios como un indicador válido porque relaciona el estado físico con el mental, está poco condicionada por las interpretaciones médicas de los síntomas y resulta un buen predictor de mortalidad (Rohlfs et al. 2000). En cuanto a la autopercepción de la salud, se observa en la ENES que un elevado porcentaje de los encuestados lo evalúan como bueno o muy bueno (74,7 $\%$ en Conurbano, $80 \%$ en CABA). Aproximadamente dos de cada diez encuestados consideraron su estado 
como regular y solo un $4 \%$ lo califica como malo. A medida que avanzan las edades de los entrevistados decrece la autopercepción favorable y aumenta sobre todo la valoración del estado de salud "regular".

La consulta sobre la manifestación de algún malestar, enfermedad o accidente en el último año muestra que dos tercios de esta población no ha padecido estos problemas. La no percepción de problemas se manifiesta más entre los residentes en CABA (74\%) que en los que habitan el Conurbano (64\%). La existencia de malestares es más notoria en las personas de mayor edad (Oddone, 2018).

La 4.ta Encuesta Nacional de Factores de Riesgo (ENFR, 2018) también indaga sobre la percepción del estado de salud de las personas mayores de 65 y más años. Con cifras similares a la ENES, crece la evaluación "regular" a medida que aumenta la edad de esta población y resulta más pronunciada entre los entrevistados de la provincia de Buenos Aires si se los compara con los de la CABA. Si se toman en cuenta todos los indicadores de deterioro de la Calidad de Vida Relacionada con la Salud (CVRS), estos también son más frecuentes en mujeres y en personas mayores de 50 años (ENFR, 2018).

\section{A MOdo DE CONCLUSión}

De las fuentes analizadas para este trabajo se extraen varias conclusiones. En primer término se pretendió visibilizar un panorama sobre el acceso a la salud y a los beneficios previsionales de la población mayor signado por persistentes desigualdades.

La edad y el sexo, además de marcar diferencias biológicas en la determinación de los problemas de salud, constituyen mediadores sociales, culturales, políticos y económicos en las experiencias diferenciadas entre individuos pertenecientes a un mismo contexto social y así se refuerza la perspectiva de curso de vida que entiende la multiplicidad y diversidad de las vejeces como dimensión troncal para los análisis sociales. Frente a un problema de salud existen diferentes respuestas, lo que refuerza el carácter multidimensional de las desigualdades. De ello se deduce que las percepciones sobre cómo se siente una persona no se limitan necesariamente al diagnóstico médico sino a cómo se sobrelleva un malestar.

Los datos de ENAPROSS permiten identificar la existencia de desigualdades según jurisdicción, sexo, edad,nivel educativo y de ingresos y trayectoria laboral entre las personas mayores. Si bien las jurisdicciones analizadas tienen un nivel de Necesidades Básicas Insatisfechas (NBI) relativamente bajo en comparación con las provincias del NOA o NEA, pueden establecerse diferencias entre el porcentaje de hogares con NBI de los 24 partidos del Gran Buenos Aires (9,2 \%) y la CABA (6 \%) que se reflejan en la situación de las personas mayores.

En segundo lugar, y en relación a las acciones públicas, puede concluirse que los dispositivos desarrollados durante el período 2016/2019 muestran una reducción en la redistribución de los ingresos de las personas mayores y un retroceso respecto a la política llevada a cabo por el gobierno anterior.

Si bien a partir de 2008 se produjo un avance significativo en la cobertura previsional al incorporarse un mayor número de beneficiarios al sistema, el monto del haber mínimo reflejó las desigualdades de ingresos y de género con un escaso reconocimiento del trabajo reproductivo y de cuidado. Con los cambios producidos en 2016, las mujeres de menores ingresos y sin inserción en el mercado de trabajo formal deberán esperar hasta los 65 años para obtener una pensión universal, cuyo haber será menor a la jubilación mínima (ya de por sí exigua).

Estas nuevas normativas tienen incidencias prácticas y materiales en las limitaciones a los ingresos, el acceso a la salud y a una vida digna. Se despliegan y se argumentan con discursos ambivalentes que incorporan nociones de derechos y reconocimiento, aunque no vayan acompañadas de una real distribución monetaria que mejore las condiciones de vida de la población mayor. Se basan en meros instrumentos técnicos similares a los que esbozan los organismos internacionales con respecto al envejecimiento activo, pero que enmascaran el contexto de una aguda crisis socioeconómica. A modo de ejemplo puede distinguirse la recomposición de los haberes jubilatorios, cuya fórmula se implementó con dos trimestres de atraso, y la limitación en el acceso 
a medicamentos. La obra social de PAMI presenta, además, serias dificultades para la prestación de servicios de calidad para tantos afiliados. En la agenda pública ha circulado un proyecto en el que se habilita al afiliado a permanecer en su obra social de origen una vez que accede al beneficio de la jubilación para descomprimir así la afiliación obligatoria al PAMI. Si bien es un derecho legítimo, habría que analizar la efectiva disposición de las obras sociales gremiales para hacerse cargo de la salud de los afiliados ya jubilados.

En tercer lugar, y a un nivel macro, el reconocimiento de derechos y la protección social de las personas mayores están ligados en nuestro país al proceso de jubilación, iniciado a mediados de siglo XX, y, por ende, a una ciudadanía vinculada al trabajo y a las leyes laborales como reconocimiento de derechos.

El futuro del sistema de previsión social es complejo, ya que la financiación proviene en gran parte de la tributación general, que es notoriamente regresiva y que se ve constantemente disminuida a causa de la reducción de aportantes producida por el elevado índice de informalidad laboral. Así, la organización del sistema de protección social, que continuará estructurado según principios contributivos de tipo tradicional, estará expuesta a los vaivenes del mercado de trabajo.

En un país signado por un fuerte endeudamiento externo, baja productividad, elevado nivel de pobreza y marcados índices de desempleo, la noción de cuidado y prevención para las personas mayores está poco instalada.

Las acciones del Estado dirigidas hacia personas mayores muestran las capacidades y los obstáculos de diferentes actores para alcanzar sus objetivos y deberían tener en cuenta, de manera sostenida, los cambios producidos en los últimos años y el reconocimiento de las necesidades de las personas mayores. Si se entiende al Estado como el organizador del bienestar y de las condiciones de vida, ese reconocimiento debería darse a nivel de la legislación sin dejar de tener en cuenta para su diseño a las familias y a las mujeres cuidadoras, ya que constituyen un vértice afectado por la problemática del cuidado. En ese sentido, resulta necesario que el Estado asuma un rol protagónico en la implementación de políticas que hagan posible el ejercicio pleno del derecho a la salud y a la previsión social.

\section{REFERENCIAS}

Adelantado Gimeno, J. (2017). Reestructuración de los Estados de Bienestar ¿Hacia un cambio de paradigma? Argumentum, 9 (2), 38-52.

Alfama, E. y Cruells, M. (2016). ¿De qué hablamos cuando hablamos de envejecimiento activo? Interpretaciones distintas, propuestas divergentes. En S. Ezquerra, M. Pérez Salanova, M. Pla y J. Subirats (eds.), Edades en transición. Envejecer en el siglo XXI (pp. 57-66). Barcelona: Planeta.

Alleyne, G.A. (2000). Prólogo. En H. Standing, El género y la reforma del sector salud (pp. 1-2). Harvard: Harvard Center for Population and Development.

Amadasi, E. y Tinoboras, C. (2016). El desafio de la diversidad en el envejecimiento. Familia, sociabilidad y bienestar en un nuevo contexto. Buenos Aires: Educa. Recuperado de http://bibliotecadigital.uca.edu.ar/greenstone/cgi-bin/ library.cgi? $=\mathrm{d} \& \mathrm{c}=$ investigacion\& $\mathrm{d}=$ desafio-diversidad-envejecimiento-familia

Barbosa da Silva J. y Berti Azevedo, M. (2002). Epidemiologia e Desigualdade: Notas sobre a teoria e a história. Revista Panamericana de Salud Pública, 12(6), 375-383.

Barradas Barata, R., Almeida, M., Valencia Montero, C. y Pereira Da Silva, Z. (2002). Desigualdades en saúde segundo genero e etnia en maiores de 14 anos de idade, Brasil. I Oficina de trabalho sobre desigualdades sociais e de genero em saúde de idosos no Brasil. Ouro Preto: Núcleo de Estudos em Saude Publica e Envelhecimento (NESPE); CENEP/FUNAPSA/Ministerio da Saude; Centro de Pesquisa René Rachou da Fundacao Oswaldo Cruz (CPQRR-FIOCRUZ); Faculdade de Medicina da Universidade Federal de Minas Gerais.

Bazo, M.T. (1992). La nueva sociología de la vejez: de la teoría a los métodos. Revista Española de Investigaciones Sociológicas, 60, 75-90.

Borrell, C. y Benach, J. (2003) Les desigualtats en la salut a Catalunya. Barcelona: Editorial Mediterrània. 
Liliana Findling, et al. Políticas sociales y desigualdades en Salud: un panorama sobre las Person...

Casals, I. (1980). Hacia una sociología de la ancianidad en España. Revista Española de Investigaciones Sociológicas, 11, 91-111.

Corin, E. (1996). La matriz social y cultural de la salud y la enfermedad. En R. Evans, M. Barer y T. Marmor (eds) $\dot{ }$ Por qué alguna gente está sana y otra no?, (pp103-145), Madrid: Editorial Díaz de Santos.

Danani, C. (1998). Los procesos que esconde la reforma de la Seguridad Social. Argentina: el caso de las obras sociales. Actas del XXI LASA International Congress, Chicago. Recuperado de http://lasa.international.pitt.edu/LASA 98/Danani.pdf

Danani, C. y Hintze, S. (2011). Introducción. En C. Danani y S. Hintze (Coords.) Protecciones y Desprotecciones: Problemas y debates de la seguridad social en la Argentina (I) (pp. 9-32). Buenos Aires: Universidad Nacional de General Sarmiento.

Danani, C. y Hintze, S. (2014). Introducción. En C. Danani y S. Hintze (Coords.) Protecciones y Desprotecciones: Problemas y debates de la seguridad social en la Argentina (II) (pp. 9-16). Buenos Aires: Universidad Nacional de General Sarmiento.

Esping-Andersen, G. (1993). Los tres mundos del Estado del Bienestar. Valencia: Alfons el Magnanim.

Findling, L. (2012). Escenarios de la desregulación de las Obras Sociales Sindicales: el desafio de los actores frente a la libre elección de los servicios de salud. Buenos Aires 1997-2004 (Tesis doctoral inédita). Universidad de Buenos Aires, Facultad de Ciencias Sociales, Buenos Aires, Argentina.

Findling, L. y López, E. (coord.) (2015). De cuidados y cuidadores: acciones públicas y privadas. Buenos Aires: Biblos Editorial.

Gastrón, L. y Oddone, J. (2008). Reflexiones en torno al tiempo y el paradigma del curso de vida. Perspectivas en Psicología, 5(2), 1-9.

González, H. y González Etkin, L. (17 de noviembre de 2017). Reforma Previsional. Un proyecto de ley inconstitucional, regresivo, que vulnera los derechos humanos de los trabajadores. Boletin Reforma Previsional, pp. 1-14.

Grassi, E. (2003). Políticasy problemas sociales en la sociedad neoliberal. La otra década infame (I). Buenos Aires: Espacio Editorial.

Gwatkin, D.R. (2000). Health inequalities and the health of the poor : what do we know? What can we do?. Bulletin of the World Health Organization: the International Journal of Public Health, 78(1), 3-18.

Huenchuan, S. y Rodríguez-Piñero, L. (2010). Envejecimiento y derechos humanos: situación y perspectivas de protección. Santiago de Chile: CEPAL.

Kunst, A. E. (1997). Cross national comparisons of socioeconomic differences in mortality. Rotterdam: Thesis Erasmus University Rotterdam.

Laslett, P. (1995). Necessary Knowledge: Age and Aging in the Societies of the Past”. En D. Kertzer y P. Laslett (Ed.) Aging in the Past Demography, Society and Old Age (pp. 3-79). Berkeley: The University of California Press.

López, E., Findling, L., Lehner, M. P. y Mario, S. (2015). Aspectos sociales del envejecimiento demográfico y los cuidados. En A. L. Kornblit, A. C. Camarotti y M. Güelman (eds.) X Jornadas Nacionales de Debate Interdisciplinario en Saludy Población. Buenos Aires: Editorial Teseo. Recuperado de https://www.teseopress.c om/jornadasnacionalessalud/chapter/aspectos-sociales-del-envejecimiento-demografico-y-los-cuidados/

Macintyre, S, Hunt, K.,y Sweeting, H. (1996). Gender differences in health: are things really as simple as they seem?. Social Science Medicine, 42(4), 617-624.

Martín Palomo, M.T. (2008). Domesticar el trabajo: una reflexión a partir de los cuidados. Cuadernos de Relaciones Laborales, 26(2), 13-44.

Messina, G. (2015). Continuidades y discontinuidades en el régimen de bienestar argentino entre 2003 y 2013 ¿Promoción de la inclusión o fragmentación de la política social? Documento de Trabajo 20, CEPED. Recuperado de http://www.economicas.uba.ar/wp-content/uploads/2016/02/CEPED-DT-20-Messina.pdf

Ministerio de Desarrollo Social y Salud. Secretaría de Gobierno de Salud (2018). Dirección Nacional de Promoción de la Salud y Control de Enfermedades Crónicas No Transmisibles. $4^{\circ}$ Encuesta Nacional de Factores de Riesgo. 
Recuperado de http://www.msal.gob.ar/images/stories/bes/graficos/0000001444cnt-4ta-encuesta-nacional-fa ctores-riesgo_2019_principales-resultados.pdf

Oddone, J. (2018). Condiciones de vida de las personas mayores. En J. I. Piovani y A. Salvia (coord.) La Argentina en el siglo XXI. Cómo somos, vivimos y convivimos en una sociedad desigual. Encuesta Nacional sobre la Estructura Social (pp. 593-624). Buenos Aires: Siglo XXI.

Organización Mundial de la Salud (2008). Subsanar las desigualdades en una generación. Resumen analítico de la Comisión sobre Determinantes Sociales de la Salud (CDSS). Ginebra: Ediciones OMS. Recuperado de file:/// C:/Users/Area\%20Salud/Downloads/9789243563701_spa.pdf

Organización Panamericana de la Salud (1999). Boletín Epidemiológico, 20(1), 1-16. Washington: OPS.

PAMI (2017). Boletín del INSSJP, 12(2882), Buenos Aires: Instituto Nacional de Servicios Sociales para Jubilados y Pensionados. Recuperado de http://institucional.pami.org.ar/files/boletines_inssjp/27-09-17.pdf

Pérez, J. (junio, 2000). Envejecimiento y esperanza de vida en salud. Trabajo presentado en XXIII Congreso de la Sociedad Española de Geriatría y Gerontología. Centre d'Estudis Demográfics, Barcelona, España. Recuperado de https://ced.uab.cat/publicacions/PapersPDF/Text174.pdf

Pla, M. y Pérez Salanova, M. (2016). Las personas mayores ¿̇de objetos a sujetos? Subjetividades e identidades. En S. Ezquerra, M. Pérez Salanova, M. Pla y J. Subirats (eds.) Edades en transición. Envejecer en el siglo XXI (pp. 141-158). Barcelona: Planeta.

Rada Schultze, F. (2018). La diversidad en el curso de la vida. Trayectorias y memorias de los y las mayores LGBT argentinos. En C. E.Henning y C. Braz (Orgs.) Gênero, sexualidade e curso da vida: Diálogos latino -americanos (pp. 111-143). Goiânia: Editora Imprensa Universitária, Universidade Federal de Goiás.

Razavi, S. (2007). The political and social economy of care in the development context. Conceptual issues, research questions and policy options. Gender and Development Programme, 3. United Nations Research Institute for Social Development. Recuperado de http://www.unrisd.org/80256B3C005BCCF9/(httpAuxPages)/2DBE6 A93350A7783C12573240036D5A0/\$file/Razavi-paper.pdf

Riera, A. (14 de abril de 2016). Regazzoni: La utilidad clínica de los medicamentos que ya no recibirán una cobertura del $100 \%$ es muy baja. Chequeado.com. Recuperado de https://chequeado.com/ultimas-noticias/regazzoni-la-u tilidad-clinica-de-los-medicamentos-que-ya-no-recibiran-una-cobertura-del-100-es-muy-baja/

Rodríguez Enríquez, C. y Méndez, F. (2013) Trabajadoras del cuidado: el caso de las trabajadoras de casas particulares: ¿Una forma peculiar de informalidad? Trabajo presentado en $11^{\circ}$ Congreso Nacional de Estudios del Trabajo, Buenos Aires, Asociación Argentina de Especialistas en Estudios del Trabajo. Recuperado de https://www.ase t.org.ar/2013/ponencias/p6_Rodriguez.pdf

Rohlfs I., Borrell, C., Anitua, C., Artazcoz, L., Colomer, C. y Escribá, V. (2000). La importancia de la perspectiva de género en las encuestas de salud. Gaceta Sanitaria, 2(14),146-155.

Roqué, M., Letchner, H., Strada, J. y De Marizani, F. (2019). Informe sobre la situación de las personas mayores. El impacto inflacionario en los Medicamentos. Buenos Aires: Centro de Estudios Políticos para Personas Mayores (CEPA) y Asociación Latinoamericana de Gerontología Comunitaria. Recuperado de https://gallery.mailchimp.com/e9c6f62a4dc825f6a9dab4e88/files/71338371-7d11-47e3-a0cd-cbd9b5b3e 118/2019.04.21_Informe_precio_de_medicamentos_Abril_2019_CEPA_CEPPEMA_ALGEC.pdf

Sala, G. (2012). Cobertura previsional, empleo y desempleo entre los adultos mayores argentinos. Revista Latinoamericana de Población, 6(11), 87-108.

Scott J. W. (1988). Gender and the politics of history. New York: Columbia University Press.

Sen, A. (2002). ¿Por qué la equidad en salud? Revista Panamericana de Salud Pública, 81(11), 5-6.

Sosa, G y Mársico, N. (27 de mayo de 2018). Recorte a los jubilados a medida del FMI. Nuestras Voces, Periodismo Ciudadano. Recuperado de http://www.nuestrasvoces.com.ar/investigaciones/recorte-a-los-jubilados-a-medid a-del-fmi/

Tamer, N. (2008). La perspectiva de la longevidad: un tema para re-pensar y actuar. Revista Argentina de Sociología, $6(10), 91-110$. 
Liliana Findling, et al. Políticas sociales y desigualdades en Salud: un Panorama sobre las Person...

\section{Notas}

1 Proyecto Ubacyt 2018/20: “Políticas de cuidado de personas mayores en Argentina, Uruguay y España. Percepción de la calidad de vida e itinerarios terapéuticos y perspectivas de los profesionales”. Directoras: Liliana Findling y Elsa López.

2 En el caso de la ENAPROSS se han procesado los datos disponibles elaborando una submuestra de las personas de ambos sexos de 60 años y más que residen en CABA y en el CBA. Con respecto a la ENES solo se han descripto algunos indicadores de salud de las personas de 60 y más años de CABA y GBA a partir del artículo de Oddone (2018), pero no se ha tenido acceso a la base de datos.

3 Si bien los demógrafos consideran que una población está envejecida cuando el porcentaje de personas de 65 años o más alcanza o supera al $7 \%$ del total de la población, con otro criterio el Plan de Acción Internacional de Viena sobre el Envejecimiento de 1982 y la Segunda Asamblea Mundial sobre el Envejecimiento de 2002 adoptan los 60 años para definir a los adultos mayores. Y dado que los programas de políticas públicas para personas mayores delimitan su inclusión a partir de de los 60 años se asume dicha edad para este artículo.

4 Por razones de espacio no se presentan todos los cuadros procesados.

\section{BY-NC-SA}

